\title{
1 Image recognition based on deep learning in Haemonchus contortus motility
} assays 1203, 50005 Hradec Králové, Czech Republic.

Correspondence:

*Corresponding Author

matousp7@faf.cuni.cz

\section{Abstract}

Poor efficacy of some anthelmintics and rising concerns about the widespread drug resistance have highlighted the need for new drug discovery. The parasitic nematode Haemonchus contortus is an important model organism widely used for studies of drug resistance and drug screening with the current gold standard being the motility assay. We applied a deep learning approach Mask R-CNN for analysing motility videos and compared it to other commonly used algorithms with different levels of complexity, namely Wiggle Index and Wide Field-of-View Nematode Tracking Platform. Mask R-CNN consistently outperformed the other algorithms in terms of the forecast precision across the videos containing varying rates of motile worms with

21 a mean absolute error of 5.6\%. Using Mask R-CNN for motility assays confirmed the common problem of algorithms that use Non-Maximum Suppression in detecting overlapping objects, which negatively impacted the overall precision. The use of intersect over union (IoU) as a measure of the classification of motile / non-motile instances had an overall accuracy of $89 \%$. 
can anticipate that this method will broaden the number of possible approaches to video analysis of worm motility. IoU has shown promise as a good metric for evaluating motility of individual worms.

\section{Author summary}

Deep Learning has made significant improvements over the past years reaching a level where models are able to successfully detect heterogeneous object instances in an image. The ability to precisely detect larvae in an image then allows the researchers to perform multiple types of analyses that work with the viability of the parasites and are not only limited to motility assays, but also include larval development tests and egg hatch tests which are common in vitro assays for diagnosis of drug resistance. Although the other types of tests were not the main subject of this study, they show the versatile applications that are at hand with the use of Deep Learning models. The most important aspect of these applications is the possibility to automate these in vitro assays and scale them, therefore allowing researchers to avoid tedious repetitive tasks and focus on activities where they provide a higher added value.

\section{Introduction}

Parasitic nematode infections present a substantial problem to human and veterinary medicine. Anthelmintics are often used as a weapon of choice to control or eliminate parasitic worms. With increasing reports of drug resistance, there is an ongoing effort put into the development of new anthelmintics $[1,2]$. In view of this, drug screening and drug testing methods are clearly necessary. A number of important human and animal nematodes were already studied in large chemical library-scale screenings, although, nearly all of the studies were aimed at well-established non-parasitic Caenorhabditis elegans because of the scientific interest and medical applications as potential human disease models [3-7]. 
Nowadays, in vitro assays can measure the effects of compounds on development, growth, behaviour, and motility. Several approaches have been pursued in attempts to deliver robust, automated assays on nematode phenotype [8-11]. The current gold standard for measuring drug effectiveness is in vitro assessment of worm motility, as measured via microscopy. The automated phenotyping from short video recordings offers a useful assay for high-throughput whole-organism phenotypic screening and avoids manual scoring. In assays with parasitic worms under experimental conditions, several challenges need to be overcome to enhance performance of motility measurements. These challenges are associated with the different worm lengths, their tendency to clump, and also the range of diverse complex patterns of movement different from that seen in C. elegans.

Focusing on veterinary importance, the parasitic nematode Haemonchus contortus, known as barber's pole worm, is a model organism often used for drug screening and studying drug resistance; due to its socio-economic importance, known genome and transcriptome [12, 13]. H. contortus infects predominantly small ruminants, it resides in abomasum where it feeds on blood. It has a direct life cycle, the third-larval stage (L3s) can be stored, and researchers commonly use L3s or in vitro fourth larval stage for motility studies. Many automated or semiautomated approaches to measure motility have already been described such as via electrical impedance [14] or infrared light beam-interference [5]. Nevertheless, for our study, we have compared various (representative) algorithms on how to analyse the video recordings in $H$. contortus with a different level of complexity in terms of model preparation as well as computational cost. Therefore, we were interested to assess if the additional complexity of the methods leads to a sufficient increase in precision, that would justify the time/performance trade-off of these methods.

The first one mentioned is an algorithm which calculates a motility index value based on measuring the standard deviation of the pixel's light intensity averaged for a number of 
frames. This Wiggle Index (WI) assay led to many "hit compounds" in screening of a large variety of compound libraries $[15,16]$. The second approach called Wide Field-of-View Nematode Tracking Platform (WF-NTP) is built on the use of the Gaussian adaptive threshold of the image frames to identify worms and was applied in model free-living nematode $C$. elegans. It was initially developed for drug screening purposes, nevertheless, it has also found applications in other studies such as detailed genetic or behavioural studies [17]. In our study, we customized the associated WF-NTP software written in Python and compared it to the last approach which involves the application of deep learning.

The advent of machine learning has revolutionized the surveying and classifying of biological data including image recognition. Large-scale computerization reduced the time that needs to be spent in the laboratory, however the rate-limiting step is the analysis and interpretation of data. In the field of computer vision, deep learning algorithms with a convolutional neural network $(\mathrm{CNN})$ architecture have made rapid advancements on a variety of image classification tasks in cell cultures [18], yet none of them have been applied to parasitic nematodes. Object-detection-based deep learning methods have also been adapted for instance segmentation. They work on the principle of predicting bounding boxes for all objects in an image and use non-maximum suppression to remove redundant bounding-box predictions. Here we used a state-of-the-art region-based CNN called Mask R-CNN [19] which predicts an object mask for each bounding box, and compared it to the WI and WF-NTP methods. The aim was to evaluate the application of the Mask R-CNN method to the field of drug discovery of anthelmintics via measuring motility of $H$. contortus, using a metric called intersection over union (IoU). 


\section{Results}

\section{Motility forecast errors}

In the manual video processing, we observed the motility and the live rate. To measure the performance of the algorithms, we used the motility rate rather than the live rate even though

103 the stillness does not implicate a dead worm. The reason for this choice was that the algorithms

104 detect movement and are not able to differentiate if the movement was caused by the object 105 itself or by an interaction with another object. The general manual motility rate was always 106 higher than the manual live rate (Supplementary Table S1). We measured the performance of 107 the individual algorithms by evaluating the errors, defined as the differences between the 108 manual processing of the file and the respective algorithm. Out of the three methods, Mask R-

$109 \mathrm{CNN}$ had the lowest mean absolute error (MAE) with a value of 5.6\%, followed by WF-NTP $110(8.76 \%)$ and the WI (14.2\%). For WI we can apply a correction to limit the maximum motility 111 value to 1 , then the capped MAE is reduced to $11.8 \%$. In terms of the bias of the predictions, 112 the WI had the lowest mean error (ME) of $-0.71 \%$ therefore slightly overestimating the motility 113 rate on average, while both WF-NTP and Mask R-CNN underestimated the motility rate with 114 values of $6.52 \%$ and $1.95 \%$, respectively (Table 1 ).

115 WI had an increasing volatility with an increase in the ratio of alive worms in the group.

116 WF-NTP and Mask R-CNN had a tendency to systematically underestimate for the motility 117 group 100 as can be seen in Fig 1 . The mean and standard deviation of motility rates per motility 118 group and algorithm is shown also in Supplementary Table S2.

120 Table 1: Motility error metrics for algorithms

\begin{tabular}{lcc}
\hline Algorithm & MAE, \% & ME, \% \\
\hline Wiggle Index & 14.2 & -0.71 \\
WF-NTP & 8.76 & 6.52
\end{tabular}




Mask R-CNN $\quad 5.61 \quad 1.95$

122 The differences between the manual processing and the respective algorithm were expressed as

123 the mean absolute error (MAE) and mean error (ME).

124

125 Fig 1: Motility rates comparisons for individual motility groups per algorithm. The number of 126 the motility group indicates the percentage of live larvae. The motility rate is visualized as box 127 plots.

\section{Instance detection performance} not detect individual instances of worms and therefore this information was not available.

132 Similarly as for the motility rate, the precision was measured based on the error term between

133 the manual counts and the respective algorithm. In terms of the size of the error, the ean absolute 134 percentage error (MAPE) was used and we observed that the error was significantly lower ( $<<$ 135 0.0001, Wilcoxon signed-rank test) for Mask R-CNN (7.6\%) than for WF-NTP (40.23\%). Both 136 algorithms had a tendency to underestimate the number of worms on average, therefore not 137 detecting all the worm instances in a video. This was confirmed by the mean percentage error 138 (MPE) with values of 5.61\% for Mask R-CNN and 40.23\% for WF-NTP (Table 2). The reported 139 MAPE and MPE for WF-NTP had the same value because all the detections underestimated

140 the total number of worms. WF-NTP underestimated the worm count similarly for all motility 141 groups, while Mask R-CNN had similar box plots in comparison to the manual counts apart 142 from underestimating the motility group 0 as shown in Fig 2. The mean error and standard 143 deviation of the differences between the manual count and the respective motility algorithm is 144 shown in Supplementary Table S3. 
146 Table 2: Worm count error metrics for algorithms

\begin{tabular}{lcc}
\hline Algorithm / Metric & MAPE, \% & MPE, \% \\
\hline WF-NTP & 40.23 & 40.23 \\
Mask R-CNN & 7.6 & 5.61 \\
\hline
\end{tabular}

148 The differences between the manual counts and the respective algorithm were expressed as the 149 mean absolute percentage error (MAPE) and mean percentage error (MPE).

Fig 2: Worm count comparisons for individual motility groups per algorithm. The number of

152 the motility group indicates the percentage of live larvae. The number of worms is visualized 153 in box plots.

\section{Mask R-CNN detection and classification precision}

The precision of detecting worm instances in a video was on average $98.6 \%$. The majority of cases where the detection was incorrect was due to debris that had a similar size and shape as a worm. For the detected worms there was an overall accuracy of $89 \%$ to correctly label them as either motile or non-motile based on their mean IoU values. In Fig 3, the

160 distribution for different IoU values clearly showed that most of misclassified cases were

161 located around the threshold 0.8, while the classification accuracy increased for values further

162 away from the threshold. The precision and recall values (Table 3) confirm that the prevalent case was the misclassification of motile worms as non-motile. 
166 Wilk test. In both cases we could not reject the null hypothesis that the data were from a normal

167 distribution with p-values 0.85 and 0.35 , respectively (Table 4).

168

169 Fig 3: The distribution for different Intersection over Union (IoU). Both (a) histogram of IoU

170 and the associated number of worms per bin classified into motile / non-motile and (b)

171 histogram of IoU and the associated probability per class (motile / non-motile) show that the

172 majority of misclassified cases were located around the $0.8 \mathrm{IoU}$.

173

174 Table 3: Classification performance metrics

\begin{tabular}{lcc}
\hline Classification & Precision, \% & Recall, \% \\
\hline Motile & 94 & 88 \\
Non-Motile & 84 & 92 \\
\hline
\end{tabular}

175

176 Precision and recall metrics for classification performance

177

178 Table 4: Normality tests for the motility rate error terms between Mask R-CNN and the manual

179 processing

\begin{tabular}{lcc}
\hline Normality test & Statistic & p-value \\
\hline D'Agostino-Pearson & 0.32 & 0.85 \\
Shapiro-Wilk & 0.97 & 0.35 \\
\hline
\end{tabular}

180

181 For both normality tests we cannot reject the null hypothesis that the data were from a normal

182 distribution. 


\section{Discussion}

The motility phenotype is often used for drug screening and drug testing of anthelmintics. In our study, we deployed and compared three algorithms to measure motility in the parasitic nematode $H$. contortus. The results showed that the performance increased with an increasing complexity of the algorithm. WI apart from requiring very little configuration and being the fastest algorithm had some limitations in comparison to the other methods; it was by design not able to detect individual instances of worms and a control group with only motile worms needed to be created in order to be able to translate the WI into a percentage for the motility rate for each video, else it was hard to interpret the numerical value of the WI. Because of these limitations and the worse precision, it would be advisable to use WI only for initial studies to quickly test experimental results as the method is the easiest to set up.

For WF-NTP, overlapping parasites had the highest impact on the decline in the detection ability, as the algorithm filters out objects that are above or below a certain pixel size. This resulted in detecting overlapping worms as a single object, where these objects were then excluded based on the size threshold. In consequence, the total number of detected instances in an image was significantly lower than the actual number obtained by manually processing the videos. Although WF-NTP struggled with detecting all instances, the classification motile / non-motile worked well, once an instance was detected.

Mask R-CNN had the lowest MAE, nonetheless similarly to WF-NTP it struggled with overlapping objects, primarily due to the fact that the algorithm uses Non-Maximum Suppression. As a result, some instances were not detected, although in contrast to WF-NTP the worm clews were not discarded as a whole. The problem of detecting overlapping objects is currently an area of active research in object detection, particularly for biological data. For instance, Böhm et al. [20] are working on algorithms that can handle overlapping objects and have had success with applying their algorithm to C. elegans. Motile worms had a higher chance 
of being detected, because they had higher variability across the frames due to their movement.

In terms of precision, the algorithm was slightly biased towards underestimating the motility rate, however the errors were normally distributed enabling us to increase the number of

211 acquired videos in order to get to a desired level of confidence and therefore successfully

212 replace manual processing.

The results confirmed that IoU can be used as a metric for determining the motility of larvae, although some modifications might be required in order to deal with slow-moving worms, such as including the total distance travelled during the video to complement the frameby-frame information. Furthermore, the IoU threshold should be considered a parameter to be optimized as it can vary based on the age of the larvae, the temperature of the environment and

218 other factors. The downside of using Mask R-CNN is that it requires a quite big amount of 219 annotated data (provided in the Data availability), which is time demanding to create and that the training runs 1-2 days on a GPU instance. can be used out-of-the-box with slight modifications to a few parameters. If we need to detect individual instances, Mask R-CNN is the best choice, although it is more time-demanding to 224 prepare the data and train the model.

\section{Materials and methods}

\section{Procurement of L3s of $H$. contortus}

Six-month-old sheep was orally infected with 8,000 infective L3s of MHco3 strain

229 (Inbred Susceptible Edinburgh, ISE) [21]. Four weeks post-infection, faecal samples were

230 collected. L3s were produced from eggs by incubating faeces in a plastic box covered with foil 231 at $27^{\circ} \mathrm{C}$ for 7 days. Then, faeces were rinsed twice in tap water which was poured into $1,000 \mathrm{~mL}$ 232 conical measuring cups in which the larvae sank to bottom. To remove dirt or dead individuals, 
the pellet of larvae was pipetted out and filtered through a $20 \mu \mathrm{m}$ sieve submerged in water (27

$\left.{ }^{\circ} \mathrm{C}\right)$. Clean L3s at a concentration of approximately 4,000 L3 per $\mathrm{mL}$ were stored in culture flasks in water at $10{ }^{\circ} \mathrm{C}$ for several months. through a $20 \mu \mathrm{m}$ sieve (for $12 \mathrm{~h}$ ), this time collecting both live larvae from the bottom of the sieve and dead individuals remaining on the sieve.

All experimental procedures were examined and approved by the Ethics Committee of the Ministry of Education, Youth and Sports (Protocol MSMT-25908/2014-9).

\section{Video processing and manual counting}

Videos from a microscope camera were recorded for a duration of 10 seconds with a

244 framerate of 20 frames per second (fps) in a quality of 2560 by 2160 pixels. The videos (.avi with MJPEG compression, $\sim 0.45 \mathrm{~GB})$ were acquired using NIS-Elements Imaging Software

live and dead larvae (100:0, 75:25, 50:50, 25:75, 0:100; denoted as motility group 100, 75, 50,

25 and 0 , respectively) in 9 replicates and they contained on average 60 worms. The live larvae were kept in $37^{\circ} \mathrm{C}$ to maintain their motility.

The video recordings were processed so that each worm was labelled with a numeric and dead phenotype was recorded. The discrepancies were dealt with as following (i) a dead worm which was moving due to interference of a motile worm was counted as motile; (ii) a live coil non-motile worm was counted as non-motile; (iii) the worms which were not labelled by 
microscopical pathologies, such as the presence of large vacuoles in the tegument and straight phenotype, were observed. among the three models.

\section{Wiggle Index analysis}

We used an ImageJ implementation of the WI for the analysis [22]. We did not apply any scaling of the videos, the Gaussian image blur was set to 2 , the number of frames to be averaged was set to 50 and the motility output was based on the standard deviation. Since the values from the WI are relative, we used motility group 100 as a control group to be able to convert these relative values to percentages. We calculated the mean value for the group 100 and divided all values by the obtained mean. However, after the conversion some values may be higher than 1 , which in the context of motility does not make sense, therefore those values can be capped to 1 for the calculation of the error metrics.

\section{WF-NTP analysis}

WF-NTP presents an automated evaluation of motility in C. elegans. For its successful implementation, we adopted the method for the H. contortus motility assay. However, as these parasites differ in their sizes as well as their movement, some modifications to the original configuration and algorithm were necessary. These modifications belonged generally to two 278 categories, where the first was a recalibration of the available input parameters of the WF-NTP method. This included aspects such as the image size, the size of the worms that we want to detect and other configurations (see Data availability). The other modifications were changes

281 in the evaluation of the conditions for worm motility and a limit on the minimum number of

282 frames that a worm needs to be detected in for it to be included in the evaluation. 
The original method relied on bends per second of individual worms to classify them as

either motile or non-motile, however as the movement of $H$. contortus is more complex, we

replaced the bends per second with an evaluation metric that is regularly used in image

the target mask and our prediction output [23]. The use of IoU allowed us to use the capabilities

of WF-NTP in terms of the detection of individual worm instances in an image and then apply

the IoU metric to determine the worm motility. The calculation of IoU was done for the same

worm instance in neighbouring frames to detect the change on a frame-by-frame basis and then

ensure that the comparison of WF-NTP and Mask R-CNN was aimed at the quality of the

detection of individual worm instances rather than the approach to determine the motility, we used the same IoU conditions for both methods.

minimum number of frames, that a worm needed to be detected in order to be included in the

tendency to either change the shape or overlap with other worms, therefore in terms of the

299 detection the individual worm can be lost for a certain number of frames and reappear later,

300 however this can lead to counting some instances multiple times. To limit these potential

301 duplicities (Supplementary Fig S1) as much as possible and not bias the total number of worms

302 detected in the video, we selected the highest number of worms detected in a given video across

303 all frames and added a buffer of $10 \%$ in order to account for situations where some worms were

304 not detected, for example due to their overlaps. This maximum number then served as a limit

305 for selecting the number of worms to be evaluated as motile or non-motile. The instances were

306 selected based on the number of frames in a decreasing order, therefore ensuring that we had 
data from enough frames to classify the worm correctly. Again, this restriction was applied to detections from Mask R-CNN as well to enable a comparison under the same conditions.

Mask R-CNN analysis model and re-training the top layer of the network allows us to have a smaller dataset for the

317 training of the model, while maintaining a good performance. We manually annotated a total 318 of 95 images and divided them into training (69) and validation (26) using the VGG (Visual Geometry Group) Image Annotator [25], these images contained three categories of objects eggs, second-stage larvae (L2) and third-stage larvae (L3), to fully use the capabilities of Mask $\mathrm{R}-\mathrm{CNN}$ which is able to do instance segmentation as well as classification of those instances.

The total number of annotated objects was around 10,400 divided into training (6,800 objects) and validation (3,600 objects). Then we ran the training on a GPU instance until the loss function showed no signs of improvement. To avoid overfitting, we used data augmentation and applied one or multiple of the following augmentations: flipping, contrast normalization, additive gaussian noise, multiplying the pixels by a number within a given interval to make the whole image lighter or darker. The final model had a mAP IoU of $64.1 \%$ on the validation set. instances of parasites and we calculated the centroid of the detected object in terms of the 
trajectories of objects based on certain conditions, in our case we restricted the distance that the centroid could move a maximum of 100 pixels in each dimension. As a result of detecting the trajectories, we were now able to identify the same parasite instance across the frames. increase in the length of the video and therefore we decided on using the full length of the

338 videos for the motility assay (Supplementary Fig S2).

\section{Error metrics}

For the evaluation of the performance of the individual algorithms we used standard

342 metrics for the quantification of the forecast precision. These metrics work with the error term,

343 which is defined as the difference between the actual and the forecasted value, where in our 344 case the actual value was obtained by manually processing the videos. Two aspects were 345 measured, the first one was the bias of the forecasts to determine if the algorithm is 346 systematically over or under-estimating, for that purpose we used ME or MPE, which both work 347 with the mean of the error term. The second aspect was the size of the error term irrespective if 348 the error term was positive or negative to compare the overall performance of the algorithms, 349 for that purpose we used MAE or MAPE, which both work with the mean absolute value of the 350 error term.

\section{$352 \quad$ Additional files}

353 Supplementary Table S1: Manual counts of motility and live rates per motility group

354 Supplementary Table S2: Motility rates per motility group and algorithm

355 Supplementary Table S3: Mean error between the manual count and the respective motility 356 algorithm 
357 Supplementary Table S4: Mean of differences in worm counts between the manual processing

358 and the respective algorithm

359 Supplementary Fig S1: Average number of worms in the videos per motility group for WF-

360 NTP and Mask R-CNN.

361 Supplementary Fig S2: The impact of the length of the video in frames on the percentage error

362 of the detected worms for Mask R-CNN.

363

\section{Abbreviations}

365 CNN: convolutional neural network; fps: frames per second; GPU: graphics processing unit;

366 IoU: intersection over union; MAE: mean absolute error; MAPE: mean absolute percentage

367 error; Mask R-CNN: region based convolutional neural network; ME: mean error; MPE: mean

368 percentage error; WF-NTP: Wide Field-of-View Nematode Tracking Platform; WI: Wiggle

369 Index

371 Competing Interests

372 The authors declare that they have no competing interests.

374 Funding

375 This work was supported by Charles University UNCE/18/SCI/012 and SVV 260550 as well

376 as by the project EFSA-CDN [CZ.02.1.01/0.0/0.0/16_019/0000841], co-funded by ERDF.

378 Authors' contributions

379 M.Ž. and L.T.N. designed this study and wrote the manuscript. M.Ž. implemented the deep

380 learning model, wrote the code, ran the statistical analyses and generated the figures. L.T.N.,

381 E.M. acquired and annotated the images used to train the deep learning model. M.Ž. and L.T.N. 
bioRxiv preprint doi: https://doi.org/10.1101/2021.12.01.470699; this version posted December $1,2021$. The copyright holder for this preprint (which was not certified by peer review) is the author/funder, who has granted bioRxiv a license to display the preprint in perpetuity. It is made available under aCC-BY 4.0 International license.

conducted the experiments and analyzed the results. P.M. managed the project. All authors contributed to literature review and critical revision of the manuscript.

\section{Acknowledgements}

We thank MVDr. Tomáš Kaňka for assisting with the sheep infection. The authors thank prof. Lenka Skálová for critical discussion and comments.

\section{References}

1. Jiao Y, Preston S, Hofmann A, Taki A, Baell J, Chang BCH, et al. A perspective on the discovery of selected compounds with anthelmintic activity against the barber's pole wormWhere to from here? Adv Parasitol. 2020;108:1-45. Epub 2020/04/16. doi: 10.1016/bs.apar.2019.12.003. PubMed PMID: 32291083.

2. Geary TG, Sakanari JA, Caffrey CR. Anthelmintic drug discovery: into the future. J Parasitol. 2015;101(2):125-33. Epub 2015/01/15. doi: 10.1645/14-703.1. PubMed PMID: 25584662.

3. Buckingham SD, Partridge FA, Sattelle DB. Automated, high-throughput, motility analysis in Caenorhabditis elegans and parasitic nematodes: Applications in the search for new anthelmintics. Int J Parasitol Drugs Drug Resist. 2014;4(3):226-32. Epub 2014/12/18. doi: 10.1016/j.ijpddr.2014.10.004. PubMed PMID: 25516833; PubMed Central PMCID: PMCPMC4266775.

4. Burns AR, Luciani GM, Musso G, Bagg R, Yeo M, Zhang Y, et al. Caenorhabditis elegans is a useful model for anthelmintic discovery. Nat Commun. 2015;6. doi: ARTN 7485 10.1038/ncomms8485. PubMed PMID: WOS:000357178100005.

5. Taki AC, Byrne JJ, Wang T, Sleebs BE, Nguyen N, Hall RS, et al. High-Throughput Phenotypic Assay to Screen for Anthelmintic Activity on Haemonchus contortus. Pharmaceuticals (Basel). 2021;14(7). Epub 2021/07/03. doi: 10.3390/ph14070616. PubMed PMID: 34206910; PubMed Central PMCID: PMCPMC8308562.

6. Elfawal MA, Savinov SN, Aroian RV. Drug Screening for Discovery of Broadspectrum Agents for Soil-transmitted Nematodes. Sci Rep-Uk. 2019;9. doi: ARTN 12347 10.1038/s41598-019-48720-1. PubMed PMID: WOS:000482564800003.

7. Pulak R. Techniques for analysis, sorting, and dispensing of C. elegans on the COPAS flow-sorting system. Methods Mol Biol. 2006;351:275-86. Epub 2006/09/22. doi: 10.1385/159745-151-7:275. PubMed PMID: 16988441.

8. Ramot D, Johnson BE, Berry TL, Jr., Carnell L, Goodman MB. The Parallel Worm Tracker: a platform for measuring average speed and drug-induced paralysis in nematodes. PLoS One. 2008;3(5):e2208. Epub 2008/05/22. doi: 10.1371/journal.pone.0002208. PubMed PMID: 18493300; PubMed Central PMCID: PMCPMC2373883.

9. Javer A, Ripoll-Sanchez L, Brown AEX. Powerful and interpretable behavioural features for quantitative phenotyping of Caenorhabditis elegans. Philos Trans R Soc Lond B Biol Sci. 2018;373(1758). Epub 2018/09/12. doi: 10.1098/rstb.2017.0375. PubMed PMID: 30201839 ; PubMed Central PMCID: PMCPMC6158219. 
bioRxiv preprint doi: https://doi.org/10.1101/2021.12.01.470699; this version posted December $1,2021$. The copyright holder for this preprint (which was not certified by peer review) is the author/funder, who has granted bioRxiv a license to display the preprint in perpetuity. It is made available under aCC-BY 4.0 International license.

10. Partridge FA, Brown AE, Buckingham SD, Willis NJ, Wynne GM, Forman R, et al. An automated high-throughput system for phenotypic screening of chemical libraries on $\mathrm{C}$. elegans and parasitic nematodes. Int J Parasitol Drugs Drug Resist. 2018;8(1):8-21. Epub 2017/12/11. doi: 10.1016/j.ijpddr.2017.11.004. PubMed PMID: 29223747; PubMed Central PMCID: PMCPMC5734697.

11. Puckering T, Thompson J, Sathyamurthy S, Sukumar S, Shapira T, Ebert P. Automated Wormscan. F1000Res. 2019;6:192. doi: 10.12688/f1000research.10767.3. PubMed PMID: 28413617; PubMed Central PMCID: PMCPMC5365223.

12. Gilleard JS. Haemonchus contortus as a paradigm and model to study anthelmintic drug resistance. Parasitology. 2013;140(12):1506-22. Epub 2013/09/04. doi: 10.1017/S0031182013001145. PubMed PMID: 23998513.

13. Preston S, Jabbar A, Gasser RB. A perspective on genomic-guided anthelmintic discovery and repurposing using Haemonchus contortus. Infect Genet Evol. 2016;40:368-73. Epub 2015/07/07. doi: 10.1016/j.meegid.2015.06.029. PubMed PMID: 26144657.

14. Smout MJ, Kotze AC, McCarthy JS, Loukas A. A novel high throughput assay for anthelmintic drug screening and resistance diagnosis by real-time monitoring of parasite motility. PLoS Negl Trop Dis. 2010;4(11):e885. Epub 2010/11/26. doi: 10.1371/journal.pntd.0000885. PubMed PMID: 21103363; PubMed Central PMCID: PMCPMC2982823.

15. Jiao Y, Preston S, Koehler AV, Stroehlein AJ, Chang BCH, Simpson KJ, et al. Screening of the 'Stasis Box' identifies two kinase inhibitors under pharmaceutical development with activity against Haemonchus contortus. Parasit Vectors. 2017;10(1):323. Epub 2017/07/07. doi: 10.1186/s13071-017-2246-x. PubMed PMID: 28679424; PubMed Central PMCID: PMCPMC5499055.

16. Preston S, Jiao Y, Jabbar A, McGee SL, Laleu B, Willis P, et al. Screening of the 'Pathogen Box' identifies an approved pesticide with major anthelmintic activity against the barber's pole worm. Int J Parasitol Drugs Drug Resist. 2016;6(3):329-34. Epub 2016/08/16. doi: 10.1016/j.ijpddr.2016.07.004. PubMed PMID: 27524698; PubMed Central PMCID: PMCPMC5196485.

17. Perni M, Challa PK, Kirkegaard JB, Limbocker R, Koopman M, Hardenberg MC, et al. Massively parallel C. elegans tracking provides multi-dimensional fingerprints for phenotypic discovery. J Neurosci Methods. 2018;306:57-67. Epub 2018/02/17. doi: 10.1016/j.jneumeth.2018.02.005. PubMed PMID: 29452179.

18. Moen E, Bannon D, Kudo T, Graf W, Covert M, Van Valen D. Deep learning for cellular image analysis. Nat Methods. 2019;16(12):1233-46. doi: 10.1038/s41592-019-0403-1. PubMed PMID: WOS:000499653100023.

19. He K, Gkioxari G, Dollar P, Girshick R. Mask R-CNN. IEEE Trans Pattern Anal Mach Intell. 2020;42(2):386-97. Epub 2018/07/12. doi: 10.1109/TPAMI.2018.2844175. PubMed PMID: 29994331.

20. Böhm A, Mayer N, Brox T. Diskmask: Focusing Object Features for Accurate Instance Segmentation of Elongated or Overlapping Objects. I S Biomed Imaging. 2020:230-4. PubMed PMID: WOS:000578080300033.

21. Roos MH, Otsen M, Hoekstra R, Veenstra JG, Lenstra JA. Genetic analysis of inbreeding of two strains of the parasitic nematode Haemonchus contortus. International Journal for Parasitology. 2004;34(1):109-15. doi: 10.1016/j.ijpara.2003.10.002. PubMed PMID: WOS:000188377300013.

22. Preston S, Jabbar A, Nowell C, Joachim A, Ruttkowski B, Cardno T, et al. Practical and low cost whole-organism motility assay: A step-by-step protocol. Mol Cell Probes. 2016;30(1):13-7. Epub 2015/09/15. doi: 10.1016/j.mcp.2015.08.005. PubMed PMID: 26365227. 
473 23. Rezatofighi H, Tsoi N, Gwak J, Sadeghian A, Reid I, Savarese S. Generalized 474 Intersection over Union: A Metric and A Loss for Bounding Box Regression. Proc Cvpr Ieee. 475 2019:658-66. doi: 10.1109/Cvpr.2019.00075. PubMed PMID: WOS:000529484000067.

476 24. Lin T-Y, Maire M, Belongie S, Hays J, Perona P, Ramanan D, et al., editors. Microsoft 477 coco: Common objects in context. European conference on computer vision; 2014: Springer.

478 25. Dutta A, Zisserman A, editors. The VIA annotation software for images, audio and 479 video. Proceedings of the 27th ACM international conference on multimedia; 2019.

480 26. van der Walt S, Schonberger JL, Nunez-Iglesias J, Boulogne F, Warner JD, Yager N, et 481 al. scikit-image: image processing in Python. PeerJ. 2014;2:e453. Epub 2014/07/16. doi: 482 10.7717/peerj.453. PubMed PMID: 25024921; PubMed Central PMCID: PMCPMC4081273.

483 27. Allan D, Caswell T, Keim N, van der Wel C. TrackPy. DOI: https://doi 484 org/105281/zenodo. 2014;9971.

485 


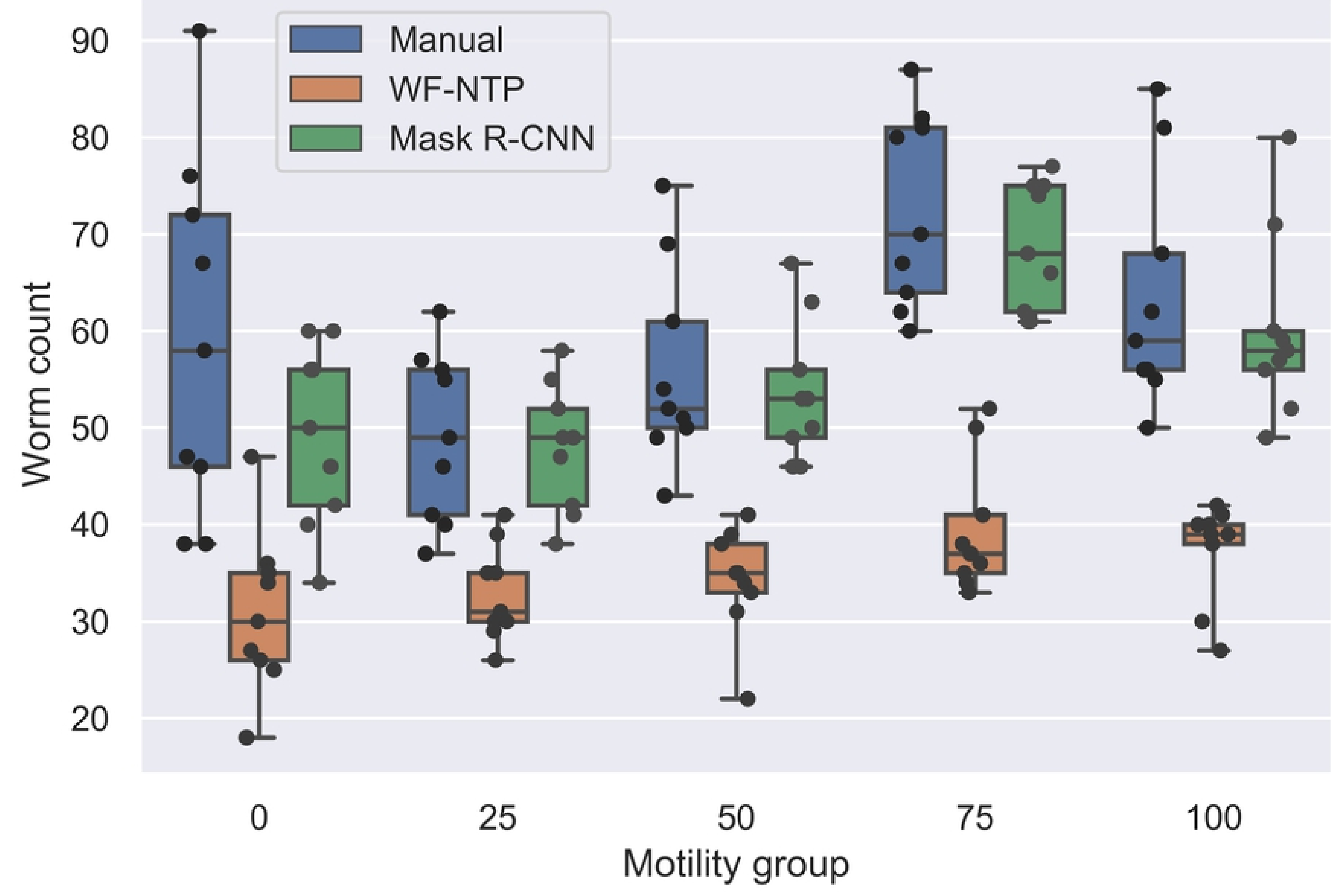

Figure 2 


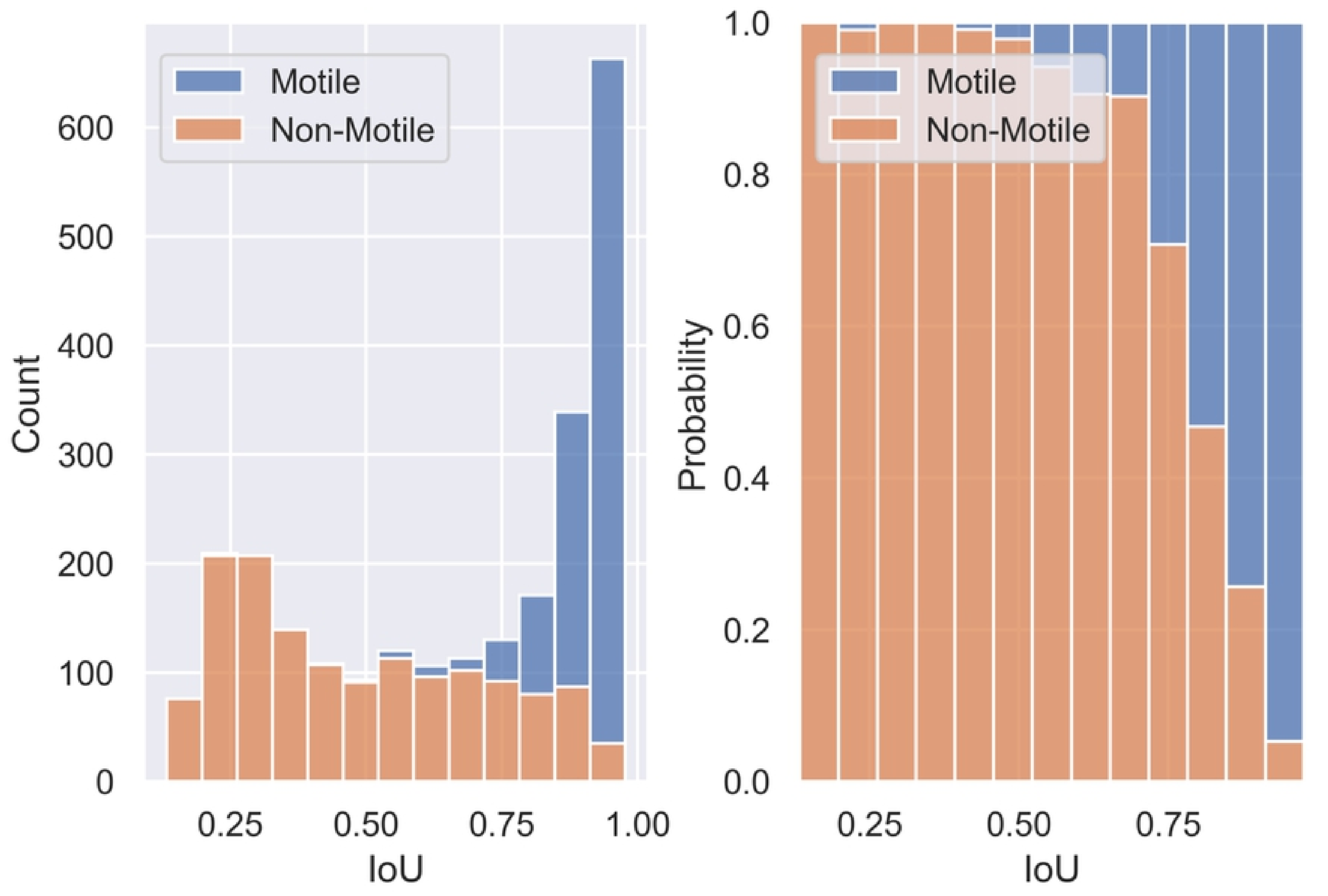

Figure 3 